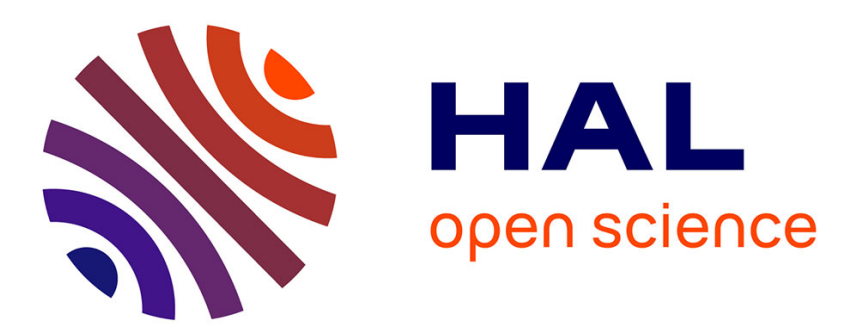

\title{
Le port de Brest et la Marine de guerre française au coeur de la pandémie de grippe infectieuse de 1918 Jean-Christophe Fichou
}

\section{To cite this version:}

Jean-Christophe Fichou. Le port de Brest et la Marine de guerre française au coeur de la pandémie de grippe infectieuse de 1918. Annales de Bretagne et des Pays de l'Ouest, 2020, 127 (2), 10.4000/abpo.5596 . hal-02922861

\section{HAL Id: hal-02922861 \\ https://hal.univ-rennes2.fr/hal-02922861}

Submitted on 26 Aug 2020

HAL is a multi-disciplinary open access archive for the deposit and dissemination of scientific research documents, whether they are published or not. The documents may come from teaching and research institutions in France or abroad, or from public or private research centers.
L'archive ouverte pluridisciplinaire HAL, est destinée au dépôt et à la diffusion de documents scientifiques de niveau recherche, publiés ou non, émanant des établissements d'enseignement et de recherche français ou étrangers, des laboratoires publics ou privés. 


\title{
Le port de Brest et la Marine de guerre française au cœur de la pandémie de grippe infectieuse de 1918
}

\author{
Jean-Christophe FICHOU, \\ professeur de géographie en CPGE au lycée de Kerichen, Brest
}

\begin{abstract}
«Toutes les fois que la guerre a nécessité le rassemblement prolongé dans nos ports militaires de grandes masses d'hommes, matelots et soldats, on y a vu survenir de grandes épidémies ${ }^{1}$ ».
\end{abstract}

Apparue à la fin de la Grande Guerre, la grippe infectieuse, dite « espagnole », peut être considérée comme un épiphénomène de celle-ci. En effet, les importants brassages de populations, les conditions d'existence très difficiles au front et sur les bâtiments de transport de troupes où régnait la promiscuité forcée des soldats et des marins, ont contribué au développement de l'épidémie. Selon l'historiographie consacrée ${ }^{2}$, la pandémie de grippe qui a sévi dans le monde en 1918 reste unique en son genre. Elle s'est produite en trois vagues distinctes: la première, printanière, très contagieuse bien que relativement douce, entre mars et août 1918 ; la deuxième, beaucoup plus sévère, de septembre à décembre 1918 et la troisième, moins intense, entre janvier et mai 1919. Bien que la durée de ces vagues ait été réduite à 14 mois dans le monde, la santé de millions de personnes en a été affectée.

La France, bien entendu, n'a pas été épargnée. Sur une durée de neuf mois, d'août 1918 à avril 1919, émergent deux pics de mortalité : I'un en septembre-octobre 1918, l'autre, de moindre importance, en février et mars 1919. Le total des décès grippaux au cours de cette période s'élève officiellement à 210900 se décomposant de la façon suivante :

- Décès civils en 1918 : 128000 (données corrigées après analyse des causes de décès)

- Décès civils en $1919: 36018$ (estimation minimale)

- Décès militaires en 1918-1919: 30382

- Décès civils par pneumonie grippale en 1918 : 43 501, alors que la moyenne des années 1915, 1916 et 1917 n'est que de 16 500. L'excédent anormal de 27000 décès peut être attribué, selon la majorité des études, à l'épidémie de grippe ${ }^{3}$. Dans tous les cas, il s'agit d'une fourchette basse car le gouvernement, d'une part, a tout fait pour minimiser les chiffres, et, d'autre part, ne dispose ni des compétences nécessaires ni des statistiques nécessaires pour effectuer un décompte précis. Une estimation plus récente aboutit au chiffre de 240000 décès civils et militaires pour l'automne de 1918, estimation sans doute plus proche de la réalité ${ }^{4}$, mais certains auteurs poussent le chiffre jusqu'à

1 HAMET, Louis, "Les Pleurésies purulentes grippales à streptocoques, leur traitement », Archives de médecine et pharmacie navales, t. 114, 1923, p. 21.

2 Crosby, Alfred, Epidemic and Peace, 1918, Westport, Greenwood, 1976, 337 p. (l'un des premiers ouvrages, sinon le premier, à traiter de la grippe " espagnole » en historien). KNIGHT, Joan, Eileen, The social impact of the influenza pandemic of 1918-19: with special reference to the East Midlands. PhD thesis of philosophy, University of Nottingham, 2015, p. 37.

3 DARMON, Pierre, "Une tragédie dans la tragédie : la Grippe espagnole en France (avril 1918-avril 1919) », Annales de Démographie Historique, 2000-2, p. 166.

4 ZYLBeRman, Patrick, "Comme en 1918 ! La grippe " espagnole » et nous ", Médecine Science, Vol. 22, n8-9, août-septembre 2006, p. 769. 
$400000^{5}$. En effet, la multiplication des recherches aboutit à une réévaluation continue de la mortalité provoquée par cette grippe.

La grippe espagnole reste la plus terrible des épidémies de la Grande Guerre au cours de laquelle est mort de maladie « le plus grand nombre » de marins ${ }^{6}$ et dans un laps de temps très court : de la fin du mois d'août 1918 à février-mars 1919. La pandémie mondiale de grippe H1N1 (grippe espagnole), est née probablement en Chine, et elle aurait muté aux États-Unis pour devenir mortelle avant de se répandre sur la planète entière. Toutefois, si les travaux, notamment anglo-saxons, sur la grippe " espagnole » restent très nombreux, la connaissance des origines de l'agent infectieux est pour le moins douteuse et fluctuante. II est impossible en particulier de dater la transmission du virus à l'homme ${ }^{7}$. Le germe de l'infection est toujours inconnu, « le lieu d'origine de l'épidémie n'a pu être exactement déterminé, les facteurs sous l'influence desquels la gravité de la maladie a présenté de grandes variations sont demeurés mystérieux ${ }^{8}$ ».

La découverte aux archives du Service Historique de la Marine de Brest ${ }^{9}$ de faits nouveaux, ne concernant que les marins admis aux hôpitaux militaires de Brest, hôpital maritime et hôpital de l'École des mécaniciens, nous prouve indubitablement que le schéma de la propagation généralement admis est à revoir ; la grippe n'est pas " née » aux États-Unis le 4 mars 1918, puisqu'elle est enregistrée et parfaitement décrite à Brest trois semaines auparavant, dès le 8 février. Dans tous les cas, ces renseignements confirment et amplifient la surmortalité des marins de la Marine militaire française.

\section{La première vague : Brest, épicentre de l'épidémie?}

Bien que l'origine de la pandémie reste " an unfinished story of discovery ${ }^{10}$ ", les médecins, épidémiologistes et les biologistes ont dès 1918, été confrontés à l'énigme médicale de cette pandémie ; les historiens se sont saisis plus tardivement du sujet, à partir de la fin des années $1970^{11}$. Pour la majorité d'entre eux ${ }^{12}$, le premier cas de grippe épidémique considéré comme tel apparaît le 4 mars 1918, au camp Fuston dans le comté d'Haskell au Kansas, où décède une jeune recrue, Albert Gitchell, puis la maladie se propage dans les autres camps militaires américains où s'entassent les soldats en partance vers l'Europe par voie maritime ${ }^{13}$. Par convention, le cas Gitchell, l'un des

\footnotetext{
5 BerChe, Patrick, « La Grippe espagnole », Biologie et Histoire, n`310, 2013, p. 66.

$6 \quad$ HUBer, Michel, La Population de la France pendant la guerre, Paris, PUF, 1931, p. 415.

7 VIET, Vincent, La Santé en guerre, 1914-1918: Une politique pionnière en univers incertain, Paris, Sciences Po, 2015, p. 551.
}

8 BERnARD, Léon, La Défense de la santé publique pendant la guerre, Paris, PUF, 1929, p. 147.

9 Service Historique de la Défense (désormais SHD) Marine, Brest, 3F475-479, Enregistrements journaliers des causes de décès.

10 BeVERIDGE, William, The last great Plague ; An Unfinished Story, London, Heinemann, 1977, p. 4.

11 VAGNERON, Frédéric, "La Grippe espagnole : une historiographie centenaire revisitée ", Ler História [Online], 2018, posto online no dia 27 dezembro 2018, consulté le 9 juin 2019.

12 VINET Freddy, La grande grippe 1918. La pire épidémie du siècle, Paris, Éditions Vendémiaire, 2018, 259 p.

13 HUMPHREYS, Margaret, "The influenza of 1918. Evolutionary perspectives in a historical context », dans Evolution, Medicine, and Public Health, 2018, p. 220 ; TAUBENBERGER, Jeffrey et MORENS, David, " 1918 Influenza : the mother of all pandemics », Emerging Infectious Diseases, 12-1, 2006, p. 15-22 ; PATTERSON, David et PYLE, Gerald, « The geography and mortality of the 
premiers certifiés, est désormais admis comme celui du début de la pandémie. Elle atteint New York dès le 11 mars et en avril, elle se diffuse aux populations civiles américaines puis elle gagne l'Europe entre avril et juillet, et continue sa diffusion à travers tout le continent en touchant l'Espagne, le Portugal, les Pays-Bas, la Norvège, la Russie, la Grèce et puis la Suisse ${ }^{14}$, sans doute propagée au départ après l'arrivée des combattants américains à Bordeaux en avril 1918, et leurs déplacements vers le front de la Somme. II est certain que l'épidémie arrive au front aux environs du 10 avril 1918 dans les tranchées à Villers-sur-Coudun, dans l'Oise ${ }^{15}$, et au sein du $66^{\mathrm{e}} \mathrm{RI}$ qui est cantonné dans la Somme ${ }^{16}$ puis à l'hôpital complémentaire de Fontainebleau, et au camp d'instruction automobile de Fère-Brianges dans la Marne. De là, elle se propage sur tout le front: elle est à Adinkerque en Belgique, le 22 avril $^{17}$. Pour la même époque, I'historien John Barry signale que les Allemands en position sur ce front de l'Ouest sont également frappés par l'épidémie en avril : il fait référence aux mémoires du général Erich von Ludendorff, commandant en chef de l'armée allemande, lequel rappelle que : "C'était une affaire grave d'avoir à écouter tous les matins le récital des chefs d'état-major du nombre de cas d'influenza ${ }^{18}$ ».

Dans tous les cas, " dès la fin avril, l'affection présente un caractère de diffusion extrême et atteint toutes les Armées ${ }^{19}$ " : elle touche les marins américains le 15 avril à Bordeaux-Bassens ${ }^{20}$, mais aussi les populations civiles urbaines ${ }^{21}$ avant de se répandre à travers toute la France, mais surtout dans les ports, et particulièrement les militaires. Á Cherbourg, par exemple, la maladie se développe au Dépôt des équipages qui reste le seul foyer épidémique permanent "d'où sont parties toutes les poussées successives ${ }^{22}$ ".

Mais se pose un problème: si la grippe est diagnostiquée au front, elle est forcément arrivée par un port, mais lequel ? Bordeaux ? ou Marseille, où les travailleurs indochinois débarquent en nombre à la fin de l'année 1915 ? ou Toulon ? ou Brest ? En fait, on n'en sait rien ${ }^{23}$. Second problème, les médecins de l'époque ne connaissent pas encore la nature du mal si bien que les causes de décès peuvent être présentées comme des broncho-pneumonies, des bronchites douteuses, des bronchites grippales, des pneumonies, des pleurésies, des pleuro-pneumonies, des congestions pulmonaires...

1918 Influenza Pandemic », Bulletin of the History of Medicine, 65-1, 1991, p. 4-21.

14 Pays où l'on compte 4000 morts entre juin et juillet.

15 Dr DeLATER, « La Grippe dans la nation armée de 1918 à 1921 », Revue d'hygiène, n45, 1923, p. 411.

16 Historique des $66^{e}$ régiment d'Infanterie - $266^{e}$ régiment d'Infanterie, pendant la campagne 1914-1918, Tours, Mame et fils, 1920, p. 12. "Malgré les pertes élevées, bien qu'il soit continuellement en butte à l'action des obus à gaz, et qu'il ait à supporter une forte épidémie de grippe, le $\left[66^{\mathrm{e}}\right]$ Régiment occupe le secteur jusqu'aux derniers jours de mai ».

17 Nolf, Pierre, SPEHL, Émile, COLARD, Armand, FIRKET, J.-C., «L'Épidémie de grippe à l'armée de campagne belge », Archives médicales belges, n¹, janvier 1919, p. 3.

18 BARRY, John, The Great Influenza, The Story of the Deadliest Pandemic in History, New York, Viking Book, 2004, p. 171.

19 Archives du Musée du Service de Santé des Armées, Paris, Val-de-Grâce, carton 814, Sous-secrétariat d'État du service de santé militaire, non daté

20 Dr DELATER, " La Grippe dans la nation armée... », art. cit., p. 413.

21 LAHAIE, Olivier, "L'épidémie de grippe dite « espagnole » et sa perception par l'armée française (1918-1919) », Revue Historique des Armées, n²62, 2011, p. 103.

22 MIRguet, Charles, "Quelques considérations sur l'épidémie de grippe du port de Cherbourg ", Archives de médecine et pharmacie navales, 1920, n¹09, p. 124.

${ }^{23}$ NoWAK, Mathieu, "Les Mystères de la grippe espagnole », La Recherche, $n^{\circ} 385,2005$, p. 52. 
autant de termes ambigus qui peuvent recouvrir une même affection et alors qu'il s'agit bien de la grippe. Par conséquent, il nous est impossible de dater et de localiser avec certitude le premier décès par grippe « infectieuse » comme on l'appelle alors, et qu'on a beaucoup de peine à diagnostiquer.

Pour autant, est-elle arrivée avec le débarquement des Américains comme on l'admet généralement? Les avis sont de plus en plus partagés, d'autant plus que les débarquements massifs des troupes américaines ont déjà eu lieu : le premier convoi arrive à Saint-Nazaire le 26 juin 1917 composé du $5^{\mathrm{e}}$ Régiment de Marines, la première unité à débarquer en France ; au Havre le premier convoi débarque le 25 juillet 1917 ; à Brest où le premier convoi arrive le 12 novembre 1917 ; ou à Bordeaux. Mais il n'est pour l'heure jamais question de grippe au sein du corps expéditionnaire et pourtant les médecins de $1^{\text {ère }}$ classe de la Marine Le Marc'hadour et Denier affirment que la première vague de grippe qui déferle sur l'Europe à la fin de l'été 1918 « a fait une première apparition à Brest » déjà le $1^{\mathrm{er}}$ avril ${ }^{24}$; elle est à l'hôpital maritime de Cherbourg le 20 avril $^{25}$; à l'hôpitalmaritime de Saint-Mandrier de Toulon à la même date ${ }^{26}$. Très rapidement elle touche tous les ports militaires français de la Méditerranée : Bizerte, Corfou, Salonique, Tarente... Les faits sont donc plus ou moins cohérents avec le premier décès de Fuston, le 4 mars, bien qu'on puisse se demander dans quelle mesure en moins de trois semaines, des recrues de ce camp du fin fond du Kansas auraient pu se retrouver aussi rapidement à Brest. De plus, les affirmations des deux médecins de la Marine sont inexactes ! On peut dire avec certitude que les premiers cas épidémiques commencent en février ! Le 8, presqu'un mois avant Gitchell, un premier marin, Henri Legrand, est hospitalisé pour le motif explicite de grippe ; le 11, ce sont 21 nouveaux marins qui sont enregistrés pour le même motif. II s'agit donc bien d'une épidémie. Au total, entre le 11 et le 21 février, on recense 35 marins, tous en poste au $2^{\text {ème }}$ dépôt des Équipage de la Flotte et tous soignés pour des symptômes grippaux ${ }^{27}$, bénins il est vrai, puisque tous les malades ressortent généralement après quelques jours passés à l'hôpital maritime. Aucun d'entre eux n'en meurt au cours de la deuxième vague. II est d'ailleurs très surprenant que les médecins Le Marc'hadour et Denier ne signalent pas ce pic épidémique de février dans leur article car ils en ont une parfaite connaissance : tous les deux sont alors en service à l'hôpital maritime de Brest depuis le $1^{\text {er }}$ janvier 1918 et ils ont signé les feuilles d'enregistrement et diagnostiqué avec certitude ces cas de grippe. Ne s'agit-il pas alors d'un mensonge par omission sciemment orchestré, d'une décision voulue par le gouvernement dans le contexte de la censure et de la circulation accrue des rumeurs, destiné à éviter de considérer le port de Brest comme l'épicentre de la pandémie ? Et c'est bien le port de Brest qui est concerné car les autres hôpitaux-maritimes ne sont pas touchés : à Toulon, en février 1918 nous comptons 27 décès, dont 8 de tuberculose, 6 de rougeole, 3 de méningite, les 10 restant étant des cas isolés (urémie, péritonite, paludisme, mal de Pott, dysenterie, pleurésie...), mais aucun cas de grippe ${ }^{28}$. Une seule certitude, cette vague de grippe a commencé ses plus grands ravages dans les ports militaires français de la Manche et de l'Atlantique, à Brest puis à Lorient, à Cherbourg puis à Toulon.

24 Le MARC'HADOUR, Henri et Denier, Albert, « Note sur une épidémie de la grippe à Brest », Archives de Médecine et de pharmacie navale, 1918, n¹06, p. 382.

25 MiRguet, Charles, « Quelques considérations... », art. cit., p. 125.

26 VALENCE, Albert, "La Grippe en armée navale pendant l'année 1918 », Archives de médecine et pharmacie navales, t. 107, 1919, p. 423.

27 SHD Marine, Brest, 3F475. Enregistrements journaliers, 19 février 1916-10 février 1919. On constate aussi qu'aucun de ces 35 marins ne meurt au cours de la seconde vague. La première vague les aurait protégés?

${ }_{28}$ SHD Marine, Toulon, 2F2-26. Décès enregistrés à I'hôpital maritime de Saint-Mandrier 1917-1918. 
La seule conclusion possible est que la première vague de grippe n'est pas arrivée en France en provenance des États-Unis. D'ailleurs, de nombreux chercheurs britanniques, s'appuyant sur les travaux du virologiste John Oxford ${ }^{29}$, considèrent aujourd'hui que le foyer primaire de cette épidémie est l'hôpital de l'immense camp militaire anglais d'Étaples, dans le Pas-de-Calais, où se côtoient plus de 100000 soldats. Les conditions sanitaires du camp, les incessants déplacements, la promiscuité des soldats vivant à plusieurs sous des tentes ou en baraquements, autant de motifs idéaux pour la transmission d'un virus respiratoire comme la grippe. Au mois de mars 1917, plusieurs dizaines de cas de "bronchites purulentes " sont diagnostiqués et bien que le terme de grippe ne soit pas employé, les symptômes décrits ressemblent à s'y méprendre à ceux de la grippe infectieuse. Pourtant, l'absence de propagation hors de ce camp britannique et surtout sa disparition avant la première vague de la pandémie ne sont pas en faveur du virus grippal recherché. Une troisième hypothèse peut être avancée, celle d'un foyer primaire chinois et d'une propagation au sein des 140000 coolies chinois envoyés en France pour suppléer les mobilisés et travailler dans les usines d'armement, les ports, les mines, les exploitations agricoles et les forêts ${ }^{30}$. Le seul problème, s'ils débarquent bien à Brest pour travailler sur le port, est qu'on ne note aucun cas de grippe parmi cette population; ils ne sont donc sûrement pas responsables de ce pic de février 1918. La mission pour recruter des travailleurs chinois est bien de retour le 2 mars, mais à Marseille, avec à son bord le médecin-écrivain Victor Ségalen ${ }^{31}$, alors qu'à l'hôpital maritime de Brest, l'épidémie est déjà enrayée. Reste alors une quatrième hypothèse soutenue par Geoffrey Rice qui évoque le fait que la pandémie a commencé au même moment en Amérique et en Chine et que, les deux groupes se réunissant pour des motifs de guerre, les deux souches de virus se seraient combinées pour produire une nouvelle souche de grippe hyper-virulente ; mais encore une fois, rien de certain ${ }^{32}$. Évoquer une cinquième hypothèse (ou plus ?) est donc parfaitement justifié. II ne reste plus qu'à retrouver le bateau qui apporte le germe à Brest en février 1918 et de connaître son port de départ.

En fait, la chronologie de la dispersion de l'épidémie est très mal connue parce que justement la maladie est jugée bénigne, "not a major killer ${ }^{33}$ ", mais elle reste particulièrement contagieuse. Toujours dans le Finistère, la Légation suisse informe le $1^{\text {er }}$ mai 1918 le ministère de l'Intérieur, de l'explosion d'une violente épidémie de grippe, laquelle aurait éclaté au camp d'internement de l'île-Longue, dans la rade de Brest à quelques milles du port militaire. La grippe apparaît avec certitude au début de l'été comme nous l'apprend la Dépêche de Brest ${ }^{34}$. De son côté, le médecin-chef Hervé prévient le sous-préfet de Brest le 11 juillet du caractère bénin de l'épidémie en ces termes:

"Une épidémie de grippe a débuté dans le camp depuis quelques semaines. Les hommes du service de garde ont été les premiers atteints (un sixième des hommes environ

29 OXFORD, John, «The so-called Great Spanish Influenza Pandemic of 1918 may have originated in France in 1916 », Philosophical Transactions of the Royal Society, Biological Sciences, 2001, t. 356, p. 1857-9.

$30 \mathrm{LI}$, Ma (dir.), Les travailleurs chinois en France dans la Première Guerre mondiale, Paris, CNRS-Alpha, 2012, 560 p.

31 VALENTIN, Michel, "Víctor Ségalen (1878-1919) : Introduction biographique », Histoire des sciences médicales, t. 13, 1979-1, p. 56.

32 RICE, Geoffrey, Black November: The 1918 Influenza Pandemic in New Zealand, Christchurch (NZ), Canterbury University Press, 2005, p. 53.

33 HUMPHREYS, Margaret, "The influenza... », art. cit., p. 220.

$34 \quad$ Dépêche de Brest, le 8 juillet 1918. 
de l'effectif). Depuis deux jours, il est entré à l'infirmerie du camp 7 internés. Cette épidémie offre les caractéristiques de la grippe classique. Le début est brusque. La température oscillant entre $39^{\circ}$ et $40^{\circ}$ le soir. La courbature est généralisée d'emblée. Dans tous les cas observés jusqu'ici la défervescence commence dès le $3^{\mathrm{e}}$ jour et la convalescence dès le $4^{\mathrm{e}}$. II est vraisemblable que l'épidémie, si elle s'étend, gardera jusqu'à la fin le caractère de bénignité. Les mesures prophylactiques prises sont l'isolement immédiat des malades et suspects, la désinfection de leur literie ».

Quelques jours plus tard, il confirme que

"l'épidémie a un caractère de bénignité. Les cas les plus graves, au nombre d'une centaine (95) ont été admis à l'infirmerie. La période fébrile de leur maladie a duré trois jours. Cinq cents autres environ, plus bénins, ont été traités à l'intérieur du camp. Deux seuls de ces malades ont fait une broncho-pneumonie grippale légère actuellement guérie. Depuis deux jours les cas semblent moins nombreux ${ }^{35} "$.

Cette première vague saisonnière est présentée comme sans gravité. La maladie se caractérise par " son explosion soudaine : frissons, courbatures, céphalée vive, et par une fièvre élevée qui dure trois à sept jours et disparaît sans laisser d'autres troubles qu'une fatigue insolite et une asthénie profonde ${ }^{36} »$. Elle est observée chaque année dans l'Armée navale, se présentant à l'état endémique au cours de l'hiver avec des poussées plus ou moins localisées mais c'est là une " affection banale, n'entraînant que rarement des complications, puisque en quatre ans, de 1914 à 1917, on n'a constaté que 8 cas mortels sur un total de $8258^{37}$ ". Des « complications pulmonaires bénignes, de loin en loin une pneumonie, une broncho-pneumonie pouvaient survenir mais, à part quelques septicémies et quelques broncho-pneumonies graves, la grippe guérissait après une convalescence souvent longue ${ }^{38} »$. Les autorités sanitaires ne s'alarment donc pas particulièrement, passent le fait sous silence et n'effectuent aucune étude épidémiologique, pas plus aux États-Unis qu'en France. D'ailleurs, la presse nationale est très peu prolixe à l'égard de l'épidémie de grippe; les articles sont quasi inexistants jusqu'à la fin du mois de mai. "Aucun article dans Le Petit Parisien, Le Matin et Le Journal, trois journaux à grand tirage. Et aucun article non plus dans Le Figaro, La Croix et L'Humanités9 ". Rien de plus dans la presse régionale en ce qui concerne la Dépêche de Brest ou L'Ouest-Éclair. Bien sûr la censure est intervenue pour minimiser la morbidité de cette épidémie, mais son innocuité est surtout le motif de cette indigence de commentaires. On ne compte qu'un seul décès par grippe parmi les marins brestois durant cette première période, le 25 février $1918^{40}$. La bénignité de l'épidémie est confirmée par la presse nationale plus tardivement. Ainsi, en juin 1918, le quotidien Le Gaulois nous apprend que :

«Cette affection - qui ne grandit pas quoiqu'on l'ait surnommée la grippe espagnole - est heureusement facile à conjurer par quelques lavages antiseptiques naso-pharyngiens et

${ }_{35}$ Cité dans CADIOU, Didier, «Les derniers mois du camp d'internement de l'île-Longue », Avel Gornog, n¹8, Crozon, juillet 2010, p. 34-50.

36 Moreau, René, « Qu'est-ce que la Grippe », Revue des Deux Mondes, mars 1955, p. 81.

37 ChAStANG, Léon, "L'Armée navale pendant la guerre », Archives de médecine et pharmacie navales, 1920, tome 110, p. 259.

38 MOREAU, René, "Qu'est-ce que la Grippe...», art. cit., p. 82.

39 BouRoN, Françoise, "La Grippe espagnole (1918-1919) dans les journaux français », Guerres mondiales et Conflits contemporains, $n^{\circ} 233,2009-1$, p. 84.

40 SHD Marine, Brest, 3F1082, Certificat des causes de décès ; Gourmelon Hervé, Jean, né le 21 février 1900 à Loperhet (Finistère), apprenti-marin au $2^{\text {ème }}$ Dépôt, mort le 25 février 1918 à I'hôpital maritime de Brest des suites d'une grippes infectieuse. 
l'absorption d'un ou deux cachets de quinine [...]. C'est la maladie de saison que nos pères appelaient tout bonnement le rhume des foins ${ }^{41}$ ».

En juillet 1918 un journaliste du Matin souligne avec emphase que :

«En France, [l'épidémie] est bénigne: nos troupes en particulier y résistent merveilleusement. Mais de l'autre côté du front les Boches semblent très touchés par elle. Est-ce le symptôme précurseur de la lassitude, de la défaillance des organismes dont la résistance s'épuise ? Quoi qu'il en soit, la grippe sévit en Allemagne avec intensité ${ }^{42}$ ».

La bénignité est corroborée par le voyage du président Poincaré à Brest où il arrive en gare le jeudi 15 août au matin. Accompagné du ministre de la Marine, Georges Leygues, il est accueilli par le vice-amiral Moreau, préfet maritime, le vice-amiral Schwerer, commandant supérieur des patrouilles de Bretagne, le contre-amiral Benoît, major général, le contre-amiral Grandclément, commandant le front de mer, le capitaine de vaisseau Carré, chef de division des patrouilles de Bretagne, le capitaine de vaisseau Escande, chef d'état-major de l'arrondissement... Après une visite du port de guerre, il se rend à l'hôpital maritime où "il traverse la salle des officiers et deux autres services ${ }^{43}$ ". II semble évident que ce déplacement gouvernemental n'aurait jamais eu lieu si la grippe avait représenté le moindre danger.

\section{La deuxième vague : Brest, interface de la diffusion de la grippe}

Bien qu'il s'agisse de la « répétition générale de la grande pandémie », la seconde vague n'est avérée qu'à la fin du mois d'août, laquelle « fauchera les individus par centaines de milliers ${ }^{44}$ ». En effet, durant le mois de juillet 1918, le nombre de cas diminue, mais les personnes malades présentent des grippes sévères, prolongées et de plus en plus mortelles. Toutefois, on observe au début de l'été une quasi-disparition des cas grippaux, sans explication, avant la survenue de la deuxième vague, la plus destructrice. La situation est donc paradoxale puisqu'on note, d'une part, la diminution du nombre total de grippés, mais, d'autre part, les cas de complications graves entraînant rapidement la mort qui sont en augmentation.

\section{Tableau 1 - Les conséquences de la grippe dans l'armée française}

\begin{tabular}{|l|c|c|c|}
\hline & Nombre de cas & $\begin{array}{c}\text { Nombre de } \\
\text { décès }\end{array}$ & $\%$ de décès \\
\hline Août & 3135 & 243 & $7,60 \%$ \\
\hline Septembre & 24282 & 2124 & $8,50 \%$ \\
\hline Octobre & 75519 & 617 & $0,81 \%$ \\
\hline Total & 102936 & 2984 & $2,90 \%$ \\
\hline
\end{tabular}

Entre août et octobre, le nombre exact de malades et de morts parmi les civils est inconnu ${ }^{45}$. De même, on ne voit ressurgir de nouveaux cas de grippe aux États-Unis qu'à la fin du mois d'août. Toutefois, elle est déjà bien présente en France si l'on s'en tient aux sources américaines, lesquelles signalent que la vague grippale gagne, en août 1918, la

\footnotetext{
$41 \quad$ Le Gaulois, le 26 juin 1918.

$42 \quad$ Le Matin, le 26 juillet 1918.

43 «Le Président de la République à Brest », L'Ouest-Éclair, le 17 août 1918.

44 DARMON, Pierre, « Une tragédie dans la tragédie... », art. cit., p. 156.

45 LAHAIE, Olivier, "L'Épidémie de grippe dite « espagnole » et sa perception par l'armée française (1918-1919) », Revue historique des Armées, n²62, 2011, p. 104.
} 
côte est, à Boston, où est installée une base militaire qui accueille au début de l'année 1918, un bataillon qui revenait de la région de Canton, où l'épidémie de grippe bénigne, mais particulièrement contagieuse, a sévi pendant l'hiver 1917-1918. II est probable que les militaires rapatriés de Chine rapportent avec eux le virus de la grippe qui, par mutation, donne celui de la grippe espagnole. Ce n'est qu'une hypothèse et la source géographique du virus à l'origine de la grippe de 1918 reste si incertaine qu'il est impossible de trancher sur sa provenance Selon les sources américaines, l'épidémie commence aux mêmes dates, dans deux autres ports simultanément : à Brest en France ${ }^{46}$ et à Freetown au Sierra Leone $^{47}$, un important port de chargement de charbon pour les bâtiments militaires ${ }^{48}$. Mais pour ce dernier, les informations sont tendancieuses dans la mesure où la grippe n'est pas apparue par hasard dans cette ville; en effet, le 15 août, le navire britannique HMS Mantua débarque plus de 170 passagers infectés par la grippe ${ }^{49}$. Á proprement parler, le foyer n'est pas Freetown, mais bien Porsmouth d'où le bâtiment a appareillé le $1^{\mathrm{er}}$ août et où la grippe est donc présente dès la mi-juillet, comme à Brest ! De Freetowon, elle atteint Dakar, le 22 août 1918, transportée par l'escadre brésilienne venant de Sierra- Leone ${ }^{50}$.

Elle ne peut donc être d'origine uniquement américaine, mais très certainement européenne. De plus, si on analyse les archives, on constate que la grippe est à Brest au $2^{\text {ème }}$ dépôt des Équipages dès le $1^{\text {er }}$ juillet où l'on constate huit décès entre le $1^{\text {er }}$ et le 28 du mois ; elle apparaît à Toulon le 24 juillet où elle touche essentiellement les marins du $5^{\text {ème }}$ dépôt : sur les 91 décès enregistrés à l'hôpital de Saint-Mandrier au mois d'août on en compte 59 dus à la grippe ${ }^{51}$.

«Dans un rapport au service de santé de Toulon, au sujet de la grippe espagnole, le docteur Ormes déclare que deux mille cas ont été signalés pendant le mois d'août. Ces deux mille cas se rapportent surtout à des militaires ; on signale seulement soixante-six décès. Actuellement le nombre des cas est réduit à huit cents. Les malades sont embarqués à bord du navire-hôpital Asie ou hospitalisés à Saint-Mandrier ${ }^{52}$ ".

Elle frappe à Lorient, au $3^{\text {ème }}$ dépôt, le 19 août ; à Rochefort, au $4^{\text {ème }}$ dépôt le 27 août ; à Cherbourg, au $1^{\text {er }}$ dépôt, le 30 août. Et tous les ports sont rapidement touchés : Bayonne, Bordeaux $^{53}$, Boulogne, Dunkerque, Lannion, La Rochelle, Le Havre, Rouen, Vannes... Puis elle se répand très rapidement, sur tous les continents et dans toute la France intérieure; elle est à Montpellier en août ${ }^{54}$ et signalée aussi « au village de Santes, commune d'Echassières, [où] vingt personnes, sur une quarantaine formant la population, ont été malades en même temps de la grippe espagnole " et dont quatre sont mortes ${ }^{55}$;

\footnotetext{
46 Brest où l'on recense 12 marins morts de la grippe entre le 9 et le 20 août.

47 SPINNEY, Laura, Pale rider, The Spanish Flu of 1918 and How It Changed the World, Londres, Jonathan Cape, 2017, 352 p.
}

48 GouzIEN, Paul, "La Pandémie grippale de 1918-1919 dans les colonies françaises ", Bulletin Mensuel International d'Hygiène Publique, 1920, t. 12, p. 686-724.

49 https://www.naval-history.net/OWShips-WW1-08-HMS Mantua.htm, consulté le 2 décembre 2018.

50 Dr LÉGER, Marcel, « La Peste au Sénégal de 1914 à 1924 », Annales de Médecine et de Pharmacie coloniales, 1926, t. 24, p. 317.

51 SHD Marine, Toulon, 2F2-26.

52 « La Grippe espagnole dans le Var », La Croix, le 4 septembre 1918.

53 GuILlaUme, Pierre, "La Grippe à Bordeaux en 1918 », Annales de démographie historique, 1978, p. 167-173.

${ }^{54}$ DARMON, Pierre, « Une tragédie dans la tragédie... », art. cit., p. 153.

$55 \quad$ Le Figaro, le 30 août 1918. 
elle est à Troyes en septembre ${ }^{56}$, à Lons-le-Saulnier, à Dole, à Vesoul en octobre ${ }^{57}$ ainsi qu'à Caen, au chantier naval de Blainville où les premiers morts sont tous des « Bretons récemment arrivés ${ }^{58}$ ", ce qui confirme notre hypothèse de foyer primaire à Brest où l'on enregistre pour le seul mois de septembre 690 entrées et 281 décès parmi les seuls marins $^{59}$ dont 33 le 6 septembre et autant le lendemain ${ }^{60}$. II est donc probable qu'en France le virus de la seconde vague est présent dès la fin du mois de juillet, dans les ports de guerre et il n'est sûrement pas spontané. Comme pour la première vague, on peut supposer qu'il provient d'une autre source que les États-Unis, par exemple d'Asie, réservoir connu du virus. II aurait pu être importé par les troupes coloniales d'Indochine ou par les ouvriers chinois et indochinois, déjà porteurs du " pneumocoque des Annamites », appelés en France pour servir dans les usines ${ }^{61}$. Dans tous les cas il est très difficile de situer avec précision le foyer primaire dans la mesure où la presse, muselée par la censure, ne peut fournir aucun renseignement : "Quand éclata l'épidémie de gr.... il nous fut interdit de donner un mot à cette maladie qui faisait tant de ravages [...]. La consigne fut de faire comme si elle n'existait pas ${ }^{62}$ ".

II est donc évident que tous les marins participent activement à l'expansion de l'épidémie, car ils voyagent dans le monde entier et ils sont les vecteurs actifs de la grippe. Aux États-Unis, le premier décès de la seconde vague est constaté à Boston. Par la suite, l'épidémie s'étend et tue le 4 septembre à la Nouvelle-Orléans; le 7 dans la région des Grands lacs ; sur les docks de New London le 12 ; à Seattle le $17^{63}$ ! Et les marins français sont impliqués dans le processus de propagation. On apprend que Louis Lichou (matricule 86948-2) quartier-maître sur la Gloire, alors que son bâtiment est en escale à New York, meurt de la grippe le 23 septembre à l'hôpital de Broocklin, où la première victime de l'épidémie est décédée le 18 septembre. Quinze jours plus tard, le 4 octobre, ce sont les matelots François Guermeur (95293-2) et Jean-François Simon (97867-2), matelots sur le croiseur cuirassé Marseillaise, qui décèdent dans le même hôpital et pour les mêmes motifs $^{64}$. Le 10 octobre, le matelot Joseph Mélennec (Camaret-1522), embarqué sur le croiseur Montcalm, succombe à son tour, toujours à l'hôpital de Broocklin et toujours des suites de la grippe. Tout comme son camarade de bord, le quartier-maître Jean Conseil (95382-2) qui subit le même sort le 16 octobre. Le premier cas avait été enregistré sur le Marseillaise le 8 septembre, le lendemain de son arrivé à New York après son service de convoi en Atlantique : "évolution normale sans complication ${ }^{65}$ ". Mais le 20 septembre, l'épidémie se propage de manière "foudroyante ». Au total, ce sont 25 marins français embarqués qui décèdent de la grippe entre le 23 septembre et le 24 octobre 1918 dans le

56 GuÉNEL, Jean, « La grippe "espagnole" en France en 1918-1919 », Histoire des sciences médicales, t. 38, $\mathrm{n}^{\circ} 2,2004$, p. 166.

57 Petit-Comtois, le 4 octobre 1918.

58 MOREL, Pierre, et QuÉTEL, Claude, « La Grippe "espagnole" de 1918 à Caen et son impact au Bon-Sauveur », Annales de Normandie, $27^{\mathrm{e}}$ année, n², 1977, p. 210.

59 HAMET, Louis, «Les Pleurésies purulentes grippales », Archives de médecine et pharmacies navales, t. 114, 1924, p. 23.

60 SHD Marine, Brest, 3F1091.

61 DARMON, Pierre, « Une tragédie dans la tragédie... », art. cit., p. 162.

62 « Impressions d'un passant », Le Populaire de Nantes, le 10 novembre 1918.

63 DENOFIO, Nancy, «The Deadliest Flu in History », https://angiesdiary.com, juin 2013. Consulté le 30 novembre 2018.

64 Fiches Mémoire des Hommes, 14-18.

65 CANDIOTTI, Antoine, «La Grippe à bord des bâtiments de la division de l'Atlantique », Archives de médecine et de pharmacie navales, t. 108, 1919, p. 44. 
port de New York. On peut se demander si les marins ont contracté le virus aux États-Unis au contact des infectés de terre comme il est admis ou si, au contraire, ils ne l'auraient pas apporté avec eux de Bretagne. En effet, l'épidémie est au plus fort à Brest dès la mi-août et la grippe est forcément présente à bord lors de la traversée vers les États-Unis !

\section{Tableau 2 - Cas présentés par les navires militaires français du 23 septembre au 24 octobre 1918}

\begin{tabular}{|c|c|c|c|c|c|}
\hline & Effectif & $\begin{array}{c}\text { Nbre de } \\
\text { cas }\end{array}$ & $\%$ & $\begin{array}{c}\text { Nbre de } \\
\text { décès }\end{array}$ & $\%$ \\
\hline Marseillaise & 619 & 484 & 78,2 & 15 & 3,1 \\
\hline Gloire & 615 & 106 & 17,2 & 4 & 3,7 \\
\hline Montcalm & 566 & 35 & 6,2 & 6 & 8,5 \\
\hline Condé & 612 & 28 & 4,6 & 0 & 0 \\
\hline Total & 2412 & 653 & 27,1 & 22 & 3,3 \\
\hline
\end{tabular}

Toujours est-il qu'à l'évidence, le fléau est bien réel et de retour en France, les marins porteurs du virus vont le transmettre à nouveau, essentiellement à Brest et dans la région ${ }^{66}$ où, dès le mois d'août 1918 , un cas de grippe est signalé parmi les troupes américaines présentes à Brest où plusieurs jeunes marins français du $2^{\text {ème }}$ dépôt en sont déjà malades ${ }^{67}$. Mais, comme pour la première vague, l'hôpital maritime de Brest a déjà enregistré ses premiers décès pour grippe depuis plus d'un mois ; la source ne peut pas être les États-Unis, même si le débarquement des soldats américains à Brest ou à SaintNazaire n'a sans doute fait qu'aggraver les choses.

Le $13^{e}$ régiment de Marines débarque le 7 août 1918 sur le port de commerce brestois avec plus de 100 soldats victimes de la grippe sur un effectif de 3000 hommes. Le 10 août, l'hôpital maritime de Brest est dépassé par le nombre des soldats hospitalisés et doit refuser d'admettre de nouveaux patients atteint par l'épidémie grippale.

« Elle battit surtout son plein au mois de septembre 1918, où l'on enregistra à l'hôpital 690 entrées et 281 décès pour grippe. Un même jour, le $1^{\mathrm{e}}$ septembre, vit 105 entrées et 30 décès. L'épidémie fit, en particulier, une terrible moisson dans la masse dense et réceptive des jeunes matelots rassemblés dans la caserne surpeuplée des équipages ${ }^{68} "$.

Le 2 octobre, La Dépêche de Brest publie, en première page, les confessions du médecin Louis Martin, sous-directeur de l'Institut Pasteur, envoyé en mission dans le port finistérien pour étudier et comprendre l'épidémie ayant frappé le $2^{\text {ème }}$ dépôt des équipages de la flotte alors que la maladie ne pouvait qu'être « peu grave », puisqu'elle n'était que la grippe ${ }^{69}$, en concordance avec l'avis de l'Institut et de l'Académie de médecine. Après sa visite de I'hôpital, l'inquiétude grandit :

« La particularité de cette grippe, a-t-il dit, est son caractère extrêmement dangereux de contagion. Elle se transmet exclusivement par contacts inter-humains. Un seul malade, formant point épidémique, est susceptible de contaminer toutes les personnes qui l'approchent. II y a donc lieu de considérer cette grippe comme une maladie éminemment contagieuse au même titre que la rougeole ".

\footnotetext{
66 Entretiens avec le docteur Vincent Le Berre, Plougastel-Daoulas, le 10 février 2018.

67 «Les Américains débarquent dans l'Ouest/ juin 1917 » dans Ouest France, Hors-série, 2017.

68 HAMET, Louis, « Les Pleurésies purulentes grippales... », op. cit., p. 22.

69 RASMUSSEN, Anne, "Dans l'urgence et le secret. Conflits et consensus autour de la grippe espagnole, 1918-1919 », Mil Neuf Cent, 2007-1, n²5, p. 173.
} 
En définitive, seules les mesures préconisées d'isolement s'avèrent fructueuses, mais la liste des décès continue de s'allonger. Le 8 octobre, on dénombre dans le camp de regroupement de Pontanézen, installé dans la banlieue brestoise, 12000 malades de la grippe, et 250 soldats en sont morts. Le même jour, l'USS-Léviathan arrive à Brest avec 10000 hommes, dont 4000 sont touchés par l'épidémie et plusieurs d'entre eux sont décédés à bord ${ }^{70}$. Le 5 octobre, dix marins ont succombé ; le 6 , on comptait 24 décès supplémentaires ; le 7 , ce sont 31 décès qui ont été constatés. Mais dans tous les cas, les recrues américaines débarquent dans un port totalement infesté par l'épidémie dont elles ne sont en rien responsables; elles contribuent seulement à renforcer les rangs des grippés et allonger la longue liste des décès.

Pour tous les observateurs de l'époque une telle attaque ne peut être que la conséquence d'une initiative de l'ennemi ${ }^{71}$, mais on essaie de se défendre. Le préfet maritime de Brest suspend tous les congés et permissions en septembre $1918^{72}$; on préconise le port d'un masque composé de plusieurs lames de gaze ${ }^{73}$; on interdit les visites aux inscrits grippés soignés à l'hôpital ; on isole les malades; on renvoie les malades dans leurs foyers ; on supprime, « pendant toute la durée de l'épidémie actuelle, toutes autorisations de transport à travers le département ou en dehors du département des malades décédés de grippe infectieuse ${ }^{74}$ ॥. La Marine commence à évacuer ses malades vers le lazaret de Trébéron et des hôpitaux complémentaires, notamment celui de Kervallon, tandis que les autorités civiles prennent d'énergiques mesures, interdisant notamment l'entrée de l'hospice civil aux familles des alités. Des affiches sont placardées dans toute la ville de Brest pour signaler "les précautions à prendre » pour éviter la diffusion de la maladie ${ }^{75}$. La mairie impose aussi d'inhumer les corps dans les 24 heures suivant le décès. La rentrée des classes est repoussée au 4 novembre dans le Finistère, le Morbihan et l'Ille-et-Vilaine ${ }^{76}$.

Les décès touchent particulièrement de très jeunes adultes, ce qui peut surprendre car ceux-ci constituent généralement la tranche d'âge la plus résistante aux grippes. Cette constatation a d'abord été expliquée par le fait que cette population, notamment pour des raisons de guerre, se déplace le plus ou vit dans des endroits comme les ateliers de l'arsenal ou le $2^{\text {ème }}$ dépôt des Équipage de la Flotte à Brest où elle côtoie de nombreuses personnes. Parmi les engagés à Brest en juillet, août et septembre 1918 l'hécatombe est encore plus marquée puisqu'on recense entre juillet et décembre 357 morts de la grippe, dont $27 \%$ ont 16 et 17 ans, $72 \%$ ont 18 ans, et trois n'ont que 15 ans, soit $94 \%$ du total ! ${ }^{77}$ Pas un seul de ces engagés n'a participé aux combats, et parmi eux, certains n'ont pas même fait une semaine de service. II semblerait que c'est le système immunitaire de ces jeunes recrues qui a trop vigoureusement réagi à ce nouveau virus en endommageant tous les organes, au point de tuer bon nombre des malades. Á la fin de l'année 1918, la

70 CROWELL, Benedict, The road to France. The transportation of troops and military supplies, 1917-1918, Tome 2, New Haven, Yale University press, 1921, p. 445. Le premier décès par suite de grippe est constaté le 3 octobre, après trois jours de mer.

71 BECKER, Jean-Jacques, « 20 millions de morts ! La grippe espagnole a frappé », L'Histoire, $\mathrm{n}^{\circ} 40,1981$, p. 82.

72 SHD Marine, Brest, 6P1-122, Brest le 25 septembre 1918, la préfecture maritime.

73 VINCENT, $H$. et LOCHON, G., " La Prophylaxie mécanique de certaines maladies contagieuses des voies aériennes », Revue d'hygiène et de police sanitaire, 1919, n41, p. 33.

$74 \quad$ Le Citoyen, le 20 septembre 1918.

75 SHD Marine, Brest, 1H36, Brest, octobre 1918.

$76 \quad$ Nouvelliste du Morbihan, le 13 octobre 1918.

77 SHD Marine, Brest, 1M324, 1M325, 1M326, $1 \mathrm{M} 327$ et $1 \mathrm{M} 328$. 
France déplore plus de 128000 décès civils ${ }^{78}$, mais une estimation plus récente aboutit au chiffre de 240000 décès civils et militaires à la fin de l'automne $1918{ }^{79}$. Avec plus de certitude, on dénombre plus de $30000^{80}$ décès aux armées du fait de la grippe et parmi ces derniers, au moins 1707 marins recensés, sans compter les décès dus à des pneumonies, des broncho-pneumonies, des congestions pulmonaires d'origine grippale..., conséquences directes de la pandémie grippale et de son pouvoir létal. Á Brest, la mortalité parmi les malades dirigés vers les hôpitaux maritimes de la ville est « de $12 \%$ environ ${ }^{81}$ » et elle frappe sans distinction de nationalité. Parmi les 214 grippés décédés à Brest dans la dernière semaine d'octobre, on compte 52 Français, 12 Hindous, 2 Anglais, 3 prisonniers de guerre allemands, 3 coloniaux et 141 Américains $^{82}$. Et personne ne sait comment enrayer cette pandémie :

«Dans l'ensemble, les mesures prophylactiques instituées contre la grippe ne semblent pas avoir exercé une influence notable sur la marche de la pandémie. Les appréciations que portent à leur sujet les Administrations sanitaires sont assez exactement résumées par la suivante, qui est celle de la réponse anglaise. Aucune des mesures de prophylaxie suggérées ou adoptées ne mérite beaucoup de confiance. Peut-être, prises en bloc, ontelles contribué quelque peu à diminuer la violence de l'épidémie et aidé les médecins, les infirmières, tous les services sanitaires à lutter contre le fléau ${ }^{83}$ ».

Cet aveu d'impuissance est symptomatique de l'incapacité du corps médical à lutter contre la grippe et toutes les mesures envisagées pour l'enrayer aboutissent à un échec, en France comme partout ailleurs dans le monde. L'étude des mesures prophylactiques prises est dominée par cette notion d'impuissance. La cause d'un insuccès si manifeste " a été notre ignorance concernant cette maladie, ignorance presque aussi foncière que celle de nos aïeux subissant des épidémies meurtrières sans en pouvoir résoudre l'énigme ${ }^{84} »$. Pour couronner le tout, le service de santé de l'hôpital maritime de Brest décide au mois d'août, des les premiers jours de l'épidémie mortelle, d'accorder des permissions aux jeunes recrues atteintes du mal, si bien que le taux de mortalité de marins morts dans leurs foyers est très élevé entre la mi-août et la mi-septembre permettant au virus une contamination encore plus large.

On constate seulement que la grande majorité des décès touche des marins très jeunes, et généralement des apprentis-marins engagés volontaires. De Brest, un soldat écrit que 450 marins de la classe 20 sont morts de la grippe espagnole ${ }^{85}$ En 1918, les nouvelles recrues ne sont plus des inscrits maritimes, mais bien des engagés dont les départements d'origine ne sont plus bretons, voire plus du tout maritimes ! II s'agit pour nombre d'entre eux de véritables stratégies d'évitement pour échapper à une éventuelle unité d'infanterie beaucoup plus mortifère, car en 1918 on connaît les chiffres des marins

78 DARMON, Pierre, « Une Tragédie... », art. cit., p. 165.

79 PATTERSON, David et PYLE, Gerald, « The Geography and mortality of the 1918 influenza pandemic », Bulletin ot the History of Medecine, 1991, n 65, p. 4-21.

80 DARMON, Pierre, "Une Tragédie... », art. cit., p. 166. II nous donne le chiffre de 30382 décès militaires, qui est le chiffre officiel fourni par le service de santé de l'Armée ; Vincent VIET, quant à lui, indique 33321 décès (La Santé en guerre..., op. cit., p. 553).

81 Le Marc'HAdour, Henri et Denier, Albert, " Note sur une épidémie... », art. cit., p. 386.

82 Archives du Service de Santé des Armées-Val-de-Grâce, Rapport du 30 octobre 1918, Médecin major Malloizel, $11^{\text {ème }}$ Région, Brest, ASSA, carton 809.

83 POTTEVIN, Henri, "Rapport sur le pandémie grippale de 1918-1919 », Bulletin mensuel de I'Office internationale d'Hygiène publique, t. 13-2, 1921, p. 179.

${ }^{84}$ BeRnARD, Léon, La Défense de la santé publique pendant la guerre, Paris, PUF, 1929, p. 145.

85 DARMON, Pierre, « Une tragédie dans la tragédie... », art. cit., p. 161. 
morts au combat et il est beaucoup plus réduit que celui des fantassins... En l'occurrence, les dégâts occasionnés par la grippe ne pouvaient pas être envisagés et pour bon nombre de jeunes engagés elle s'avère dévastatrice.

\section{Tableau 3 - Statistiques militaires des décès jusqu'à l'armistice selon C. Dopter ${ }^{86}$}

\begin{tabular}{|l|c|c|c|c|}
\hline & $\begin{array}{c}\text { Nbre de } \\
\text { cas }\end{array}$ & $\begin{array}{c}\text { Nbre de } \\
\text { morts }\end{array}$ & $\%$ & $\begin{array}{c}\text { dont morts } \\
\text { à Brest }\end{array}$ \\
\hline Juillet & 2950 & 6 & 0,2 & 8 \\
\hline Août & 3153 & 243 & 7,7 & 55 \\
\hline Septembre & 24282 & 2124 & 8,7 & 281 \\
\hline Octobre & 75719 & 6017 & 7,9 & 41 \\
\hline Total partiel & 106104 & 8390 & 7,9 & 385 \\
\hline $\begin{array}{l}\text { Novembre } \\
\text { (décade avant } \\
\text { l'armistice) }\end{array}$ & 16383 & 1240 & 7,7 & 3 \\
\hline Total & $\mathbf{1 2 2 ~ 4 8 7}$ & $\mathbf{9 6 3 0}$ & $\mathbf{7 , 8}$ & $\mathbf{3 8 8}$ \\
\hline
\end{tabular}

\section{La troisième vague : une lente décrue mortifère et la fin de l'épidémie}

Si l'on observe un répit au mois de novembre, on constate l'arrivée d'une troisième vague dès la fin du mois de décembre. De nouveau, les ports sont les premiers touchés, notamment les équipages de navires commerciaux et militaires. Á Brest, de décembre 1918 au printemps 1919, l'épidémie entraîne dans la mort de nombreux marins. Elle touche notamment l'armée américaine, qui compte plusieurs milliers de morts, le deuxième dépôt des Équipages et le quartier de Recouvrance à Brest. La vague de grippe $^{88}$ qui déferle sur Brest d'août 1918 à mai 1919 entraîne 2825 entrées de marins et soldats français dans les hôpitaux maritimes et cause 471 décès, dont 418 marins recensés avec certitude, soit une mortalité de 1 pour 6 .

Cette troisième et dernière vague de la pandémie se renforce au cours de l'hiver 1918-1919 ; toutefois, elle ne s'est pas présentée dans tous les pays et le recensement de son passage est moins bien défini dans la littérature historique. II convient encore une fois de se méfier des affirmations des auteurs qui considèrent la deuxième vague en voie d'extinction en France dès la fin du mois de novembre 1918 : on compte 48 décès parmi les marins brestois entre le 29 novembre et le 21 décembre ${ }^{89}$. Toujours est-il que la troisième vague est apparue à la fin du mois de décembre 1918 aux EUA où elle atteint un pic à New York à la fin du mois de janvier, comme en Australie, et à l'hôpital maritime de Toulon où elle réapparaît le 29. Date à laquelle elle a déjà tué 5 marins français à Constantinople. Le mois suivant, elle se répand dans toute la Grande-Bretagne, et ensuite dans toute l'Europe. Elle est à Paris en février pendant les négociations de paix ${ }^{90}$, à Copenhague où sont morts 3 marins français ; puis, avec la démobilisation et le retour des troupes à la fin de la guerre, elle atteint des territoires éloignés de l'Europe. Ce nouveau

${ }^{86}$ DOPTER, Charles, Les maladies infectieuses pendant la guerre. Étude épidémiologique, Paris, Félix Alcan, 1921, 307 p. Statistiques qui s'avèrent inférieures à la réalité.

${ }_{87} \quad$ Selon le dépouillement des séries SHD Marine Brest, 3F1082, 1M324 et 1M325

88 HAMET, Louis, «Les Pleurésies purulentes grippales... », art. cit., p. 22.

89 SHD Marine, Brest, 3F1090, Certificat d'enregistrement de procès-verbaux de mises en bière, mai 1917-mai 1918 et 3F1091, Enregistrement des décès, décembre 1917-décembre 1919.

90 SPINNEY, Laura et SIRIGNANO, Patrizia, La Grande Tueuse : Comment la grippe espagnole a changé le monde, Paris, Albin Michel, 2018, 
brassage très important de population facilite la propagation, notamment en Inde, en Afrique du sud, en Australie, en Nouvelle-Zélande, à Madagascar et à Tahiti où la grippe espagnole arrive à Papeete par le vapeur Navua, le 16 novembre 1918 ; le virus tue plus de 3000 personnes, soit le quart de la population. Á La Réunion, 1603 poilus rescapés de la Grande Guerre débarquent le 31 mars 1919 au port de la Pointe des Galets, rapatriés de Marseille par le paquebot à vapeur Madonna de la compagnie CyprienFabre $^{91}$; ils sont à l'origine de la grippe sur l'île. En revanche, les cas de décès parmi les marins brestois sont beaucoup plus rares : 8 en janvier 1919, 30 en février, 4 en mars...

Après les deux vagues précédentes, la grippe était déjà largement répandue avant le début officiel de la troisième vague. Certes, cette épidémie semble moins virulente ; il faut souligner que les deux vagues précédentes, dont les effets sont bien connus, permettent de savoir à quoi s'attendre et les mesures prises pour la combattre sont déjà en place et simplement poursuivies ou appliquées de nouveau, en particulier l'isolement. Mais face à la grippe, les médecins n'ont toujours aucun remède efficace à proposer. Les hôpitaux répandent davantage le virus qu'ils ne sauvent des vies. Toujours est-il que la pandémie faiblit à la fin de l'hiver. Au mois de mai 1919, on considère en Europe, que l'épidémie s'éteint, le nombre de morts est devenu négligeable. Elle est totalement étouffée en 1920, sans explication non plus. Les derniers marins morts aux hôpitaux maritimes de Toulon et de Brest le sont respectivement le 30 avril et le 6 juin $1919^{92}$.

Au total, ce sont plus de 1730 marins militaires qui sont morts de la grippe entre juillet 1918 et mai 1919, dont 471 engagés et inscrits à Brest. Si l'on considère les derniers chiffres connus des marins tués au cours de la Grande Guerre, soit environ 20 550, on constate que la pandémie grippale est responsable de la mort de $8,5 \%$ des inscrits et engagés, terrible bilan si on le compare au pourcentage de décès grippaux pour l'ensemble des armées, à peine 2,2 \%.

\section{Tableau 4 - Marins morts au cours de la guerre (août 1914-24 octobre 1919) ${ }^{93}$}

\begin{tabular}{|lr|}
\hline Fusiliers, canonniers et batteries fluviales & 3350 \\
\hline Disparus au combat en mer & 6200 \\
\hline $\begin{array}{l}\text { Morts en mer, maladies, accidents et } \\
\text { blessures (dont aéronautique maritime) }\end{array}$ & 6400 \\
\hline Sous-total & 15950 \\
\hline & \\
\hline Prêtés à la Guerre & \\
\hline Commerce et pêche & 2700 \\
\hline Total général & 1900 \\
\hline
\end{tabular}

Une dernière remarque pour signaler la grande mansuétude du gouvernement et de la Marine envers les hommes qui la servaient. Tous les marins décédés sont déclarés morts en service commandé d'une maladie épidémique et bénéficient de la mention « mort pour la France » ; leurs veuves et orphelins ont droit à une pension. II n'en a pas toujours

91 GaüZĖre, Bernard Alex et AUBRY, Pierre, "La Pandémie de grippe espagnole de 19181919 à la Réunion », Médecine et Santé Tropicales, n²5, 2015, p. 15.

92 Fiches Mémoires des Hommes. Cette base a été constituée par la numérisation et l'indexation des fiches élaborées au lendemain de la Première Guerre mondiale par l'administration des anciens combattants et aujourd'hui conservées par le ministère de la Défense.

93 Recherches effectuées par les soins de l'auteur à partir du dépouillement des registres matricules et des registres des malades de l'hôpital maritime de Brest.

${ }^{94}$ Et la liste est loin d'être complète puisqu'on peut évaluer les pertes à plus de 5000 hommes. 
été ainsi pendant le conflit pour les marins morts d'autres maladies, notamment de la tuberculose.

L'Histoire a vite oublié ce triste événement ; pourtant la pandémie de 1918 a été la plus dévastatrice de l'histoire du $X X^{e}$ siècle. La pandémie grippale de 1918-1919 et ses 40 à 50 millions de morts dans le monde selon l'Institut Pasteur, 100 millions selon des réévaluations récentes de l'OMS, mais sans doute plus, reste l'un des plus grands fléaux que l'humanité eut à affronter. Du conflit, on ne retient que l'armistice signé à 5 heures 15 en ce matin du 11 novembre 1918 et la liesse populaire qui s'ensuit. II n'empêche que l'histoire précise de cette pandémie reste à écrire, car de nombreuses zones d'ombre, des incohérences flagrantes, des anomalies avérées persistent, notamment en ce qui concerne les dates et lieux de départ de l'épidémie, connue sous la seule appellation d'infectieuse par les marins français.

La pandémie de 1918 s'est singularisée à la fois par l'extrême pouvoir pathogène de la souche, mais aussi par la tranche d'âge de ses victimes, les 20-35 ans, et même les 16-20 ans en ce qui concerne les marins français, phénomènes qui ne se sont jamais répétés jusqu'ici. Cette grippe n'a pas été une conséquence directe de la guerre, mais il est évident que les grandes concentrations de soldats et les déplacements massifs d'un continent à l'autre ont joué un rôle dans sa propagation et sa létalité. C'est surtout la très forte croissance des échanges, des communications par rail et par mer entre les cinq continents, entre le front et l'arrière, qui est à l'origine de l'ampleur de la pandémie. La multiplication des transports en tout sens a provoqué une sorte d'« égalisation bactérienne " entre catégories sociales et régions du monde et ce sont les marins, les plus impliquées dans ces échanges et vivant dans des milieux confinés et encombrés à terre comme en mer, qui en ont payé le prix fort. Les ports sont les portes d'entrée du fléau et les marins sont toujours les premiers touchés. Le nombre de décès dans cette arme s'avère aussi, relativement, beaucoup plus élevé.

Toute l'ambition de cet article est simplement de compléter les nombreuses informations sur le sujet en présentant un fait nouveau : à l'origine et au cœur de la pandémie on retrouve le port de Brest, berceau de l'infection et dont le rôle essentiel n'a jamais été étudié. Mais nous ne savons toujours pas quel fut le foyer primaire. 